\title{
ON SOME EMBEDDING THEOREMS FOR INVERSE SEMIGROUPS
}

\author{
L. O'CARROLL
}

\begin{abstract}
A semilattice decomposition of an inverse semigroup has good internal mapping properties. These are used to give natural proofs of some embedding theorems, which were originally proved in a rather artificial way.
\end{abstract}

The reader is referred to [1] for the basic theory of inverse semigroups.

In an earlier paper [3] we proved the following embedding result:

(1) An $E$-unitary inverse semigroup is isomorphic to an inverse subsemigroup of a semidirect product of a semilattice and a group.

The class of over-semigroups mentioned in (1) will be denoted by $C_{1}$. The proof of (1) used McAlister's $\boldsymbol{P}$-theorem [2]. Later, in [4], we generalised the $\boldsymbol{P}$-theory to arbitrary inverse semigroups and proved a general embedding theorem. This result was then used in [6] to prove the following generalisation of (1):

(2) A strongly $E$-reflexive inverse semigroup (i.e. one which is a semilattice of $E$-unitary inverse semigroups) is embedded in a strong semilattice of inverse semigroups each of which is a member of $C_{1}$.

The class of over-semigroups mentioned in (2) will be denoted by $\bigodot_{2}$.

Finally, in [7], (2) was used to prove the following result:

(3) An inverse semigroup is strongly $E$-reflexive (if and) only if it is a subdirect product of $E$-unitary inverse semigroups with zero added possibly.

Now a member of $C_{1}$, with zero added possibly, is a member of $C_{2}$ in a rather trivial way. Moreover, it is relatively easy to see that $e_{2}$ is closed under arbitrary direct products. From this it follows that not only can (3) be deduced from (2), but that (2) can be deduced from a combination of (1) and (3).

The proof of (1) in [3], which is based on the original 'external' or 'abstract' $P$-theorem [2], involves the intuitively plausible idea of suitably completing the semilattice component of a $\boldsymbol{P}$-triple so that the group component can act on it without constraint (see [3] for details). Meanwhile, Schein [9] had given a succinct and conceptually clear proof of the $P$-theorem, which, as we shall see, gives (1) as an almost immediate byproduct. In [5] we showed that Schein's 'internal' approach could be successfully adapted to give the generalized $\boldsymbol{P}$-theory and to prove the generalised embedding theorem.

In contrast to this near ideal state of affairs for the $E$-unitary case, the general embedding theorem (and so its consequences (2) and (3)), whether in 'external' or

Received by the editors September 29, 1980.

1980 Mathematics Subject Classification. Primary 20M10.

(C) 1981 American Mathematical Society 0002-9939/81/0000-0401/\$01.75 
'internal' form, has always seemed rather mysterious. The proof proceeds by blind generalisation of the $E$-unitary case, and all that can be said in its favour is that it works and has useful consequences.

In a recent paper [8], it was noted that a semilattice decomposition of an inverse semigroup has certain good internal mapping properties. (In fact it was shown that more general " $D$-saturated unions" have these good properties, and all that follows below could be generalised to $\mathscr{Q}$-saturated unions and the $L$-semigroups of [5]; for the sake of explicitness we confine ourselves to semilattice decompositions and $E$-unitary inverse semigroups so as to apply ourselves directly to (2) and (3).) We develop this approach here so as to give what we hope is a conceptually clear, internal, and relatively concrete proof of an amalgam of (1) and (3). In view of our previous remarks, this will then put (2) on an equally satisfactory footing.

It turns out that once we have set things in the right perspective, and defined the appropriate maps, there is very little new in the way of proof needed. The algebra required is already contained in the proof of [9, Lemma 2] in immanent form.

To get down to details, let $U=\cup\left\{U_{\lambda}: \lambda \in \Lambda\right\}$ be a semilattice decomposition of an inverse semigroup $U$. Choose a particular element $\lambda_{0} \in \Lambda$, and denote $U_{\lambda_{0}}$ by $S=S\left(\lambda_{0}\right)$. Let $R=R\left(\lambda_{0}\right)=\cup\left\{U_{\lambda}: \lambda \geqslant \lambda_{0}\right\}$; note that $S$ is an ideal of $R$. Suppose furthermore that $S$ is $E$-unitary. Let $E$ denote the semilattice of idempotents of $S$, let $G$ denote the maximal group homomorphic image of $S$, and let $\phi$ : $S \rightarrow G$ be the canonical homomorphism. From [8] we see that $\phi$ lifts to a homomorphism from $R$ to $G$, which we shall also denote by $\phi$. Let $P$ denote the semilattice of subsets of $E \times G$, under intersection. Following [9], while using slightly different notation, we note that the natural action of $G$ on $E \times G$ from the left (where $g \cdot(e, h)=(e, g h)$ ) extends to a natural action of $G$ on $P$ by automorphisms (from the left). Moreover, the map $\alpha: s \rightarrow\left(s^{-1} s, s \phi\right)$ from $S$ to $E \times G$ is injective and extends to an injection of the semilattice of subsets of $S$ (under intersection) into $P$; this map will also be denoted by $\alpha$. The results of [9] can be interpreted as stating that the map $\psi=\psi\left(\lambda_{0}\right): s \mapsto((s S) \alpha, s \phi)$ is an isomorphism from $S$ onto an inverse subsemigroup of $V=V\left(\lambda_{0}\right)$, where $V$ denotes the semidirect product of the semilattice $P$ with the group $G$. (This yields (1) immediately.)

We now show that $\psi$, as well as $\phi$, has a lifting from $S$ to $R$.

THEOREM. The injective homomorphism $\psi: S \rightarrow V$ lifts to a homomorphism $\chi=$ $\chi\left(\lambda_{0}\right): R \rightarrow V$, where $\chi$ is defined by the rule

$$
r \chi=((r S) \alpha, r \phi), \quad r \in R .
$$

Remarks. Since $r S \subseteq S$ for each $r \in R$, the definition of $\chi$ makes sense. By way of motivation note first of all that $\chi \mid S=\psi$. Next, for $r \in R, r S=r r^{-1} S$, since $S$ is an ideal of $R$, and $\left(r r^{-1} S\right) \alpha$ is the union of $\left\{\left(r r^{-1} e S\right) \alpha: e \in E\right\}$; moreover, $\left\{r r^{-1} e\right.$ : $e \in E\}$ is the set of lower bounds for $r r^{-1}$ in $S$. Hence $(r S) \alpha=\left(r r^{-1} S\right) \alpha$ is the least upper bound in $P$ for the images in $P$ of the copies $r r^{-1} e S$ of these lower bounds $r r^{-1} e, e \in E$; thus $(r S) \alpha$ is a good 'deformation' of $r r^{-1}$. Alternatively, $(r S) \alpha$ is the image under $\alpha$ of the right ideal generated (in $S$ ) by these lower bounds, and the statement of [5, Theorem 3]-here we need only the case where $S$ is $E$-unitary i.e. 
the $T$ of [5, Theorem 3] is a group-shows the crucial rôle played by right ideals in building up a suitably large completion of $E$ so as to yield the desired embedding.

Proof of Theorem. Since $S$ is an ideal of $R$, the definition of $\chi$ makes sense, and $\chi \mid S=\psi$. Again, in the light of $S$ being an ideal of $R$, the latter half of the proof of [9, Lemma 2] actually shows that $(s S) \alpha \cap(s \phi) \cdot(t S) \alpha=(s t S) \alpha$, where $s$, $t$ now come from $R$. (In the original proof, $s$ and $t$ lie in $S$-of course there is no mention of an $R$-the action of $\phi$ is denoted by a bar, and the action of $\alpha$ is denoted by a hat. Note that there are two obvious minor misprints on line 1 of p. 187 there.) It follows almost immediately that $\chi$ is a homomorphism.

We can now proceed as usual. Suppose that $S\left(\lambda_{0}\right)$ is $E$-unitary, for each $\lambda_{0} \in \Lambda$. Since $R\left(\lambda_{0}\right)$ is a cone in $U, \chi\left(\lambda_{0}\right)$ extends to a homomorphism of $U$ into $V\left(\lambda_{0}\right)$ with zero added possibly, where the elements of the complement of $R\left(\lambda_{0}\right)$-if any-are sent to zero. As $\lambda_{0}$ runs through $\Lambda$, it is clear that these extended homomorphisms separate the elements of $U$, since their restrictions $\psi\left(\lambda_{0}\right)$ are injective. Hence $U$ is embedded in the direct product of the $V\left(\lambda_{0}\right)$ with zero added possibly, and (2) and (3) follow.

\section{REFERENCES}

1. J. M. Howie, An introduction to semigroup theory, Academic Press, London, 1976.

2. D. B. McAlister, Groups, semilattices and inverse semigroups. II, Trans. Amer. Math. Soc. 196 (1974), 351-370.

3. L. O'Carroll, Embedding theorems for proper inverse semigroups, J. Algebra 42 (1976), 26-40.

4. ___ Inverse semigroups as extensions of semilattices, Glasgow Math. J. 16 (1975), 12-21.

5. I_ Idempotent determined congruences on inverse semigroups, Semigroup Forum 12 (1976), 233-243.

6. ___ Strongly E-reflexive inverse semigroups, Proc. Edinburgh Math. Soc. 20 (1976-77), 339-354.

7. ___ Strongly E-reflexive inverse semigroups. II, Proc. Edinburgh Math. Soc. 21 (1978), 1-10.

8. 352-354.

9. B. M. Schein, A new proof for the McAlister P-theorem, Semigroup Forum 10 (1975), 185-188.

Department of Mathematics, The King's Buildings, Edinburgh EH9 3JZ, Scotland 